\title{
Decentralized finance
}

\author{
Katarina Frajtova Michalikova ${ }^{1, *}$ Adela Poliakova ${ }^{1}$ \\ ${ }^{1}$ University of Zilina, Faculty of Operation and Economics of Transport and Communications, \\ Department of Economics, Univerzitna 1, 01026 Zilina, Slovakia
}

\begin{abstract}
Research background: Its simplest form, decentralized finance is a system by which financial products become available on a public decentralized blockchain network, making them open to anyone to use, rather than going through middlemen like banks or brokerages. Unlike a bank or brokerage account, a government-issued ID, Social Security number, or proof of address are not necessary to use DeFi.

Purpose of the article: The aim of the contribution is more specifically, DeFi refers to a system by which software written on blockchains makes it possible for buyers, sellers, lenders, and borrowers to interact peer to peer or with a strictly software-based middleman rather than a company or institution facilitating a transaction.

Methods: Multiple technologies and protocols are used to achieve the goal of decentralization. For example, a decentralized system can consist of a mix of open-source technologies, blockchain, and proprietary software. Smart contracts that automate agreement terms between buyers and sellers or lenders and borrowers make these financial products possible. Regardless of the technology or platform used, DeFi systems are designed to remove intermediaries between transacting parties.

Findings \& Value added: The article provides an overview of decentralized finance (DeFi) solutions that have already proved they are better alternatives to traditional finance. With DeFi, users can take advantage of lower transaction rates, higher interest rates or an opportunity to diversify investments.
\end{abstract}

Keywords: decentralized finance; financial products; blockchain; globalization

JEL Classification: $G 21 ; G 33 ; M 29$

\footnotetext{
*Corresponding author: katarina.michalikova@,fpedas.uniza.sk
} 


\section{Introduction}

The use of technology in financial services is not new. Most transactions at banks or other financial services companies are accomplished with the help of technology nowadays. However, the role of technology is restricted to being a facilitator of such transactions (Kudlac et al., 2017; Majercak et al., 2013). Companies still have to contend with navigating the legalese of jurisdictions, competing financial markets, and different standards to make a transaction possible. With its stack of common software protocols and public blockchains to build them on, DeFi places technology at the front and center of transactions in the financial services industry.

DeFi is commonly placed in the domain of blockchain and cryptocurrencies. But its scope is much wider. To understand the thought processes that led to the development of decentralized finance, it is important to understand the current state of the finance ecosystem.

Modern financial infrastructure is built on a "hub and spoke" model. Key economic centers of activity, such as New York and London, function as operational hubs for the financial services industry and influence economic activity at spokes-regional centers or financial powerhouses like Mumbai or Milan that may not be as globally important as hubs but still function as nerve centers for their respective economies.

Economic prosperity or hardship radiate outward from hubs to spokes and toward the rest of the global economy. This model of interdependency is repeated in the functioning of global financial services corporations. They have headquarters in hubs and local branches, partnerships, or investments across the world. The sprawl of their operations means that the organization itself is subject to a phalanx of laws and regulations in each of its financial jurisdictions. Their reach has made such institutions systemically important to maintain the global economy's balance and necessary to maintain or create new financial services infrastructure.

Though this model worked well in the last century, the financial crisis and, subsequently, the Great Recession, revealed the flaw in this architecture. The balance sheet problems for a couple of large financial institutions produced a domino effect of tumbling economies and the onset of the global recession.

Decentralized finance uses technology to disintermediate centralized models and enable the provisioning of financial services anywhere for anyone regardless of ethnicity, age, or cultural identity. DeFi services and apps are mostly built on public blockchains, and they either replicate existing offerings built on the rails of common technology standards or they offer innovative services custom-designed for the DeFi ecosystem. At the same time, DeFi applications provide users with more control over their money through personal wallets and trading services that explicitly cater to individual users instead of institutions.

\section{Methodology}

At a broad level, the components of DeFi are the same as those for existing financial ecosystems, meaning they require stable currencies and a wide variety of use cases. DeFi components take the form of stablecoins and services like crypto exchanges and lending services. Smart contracts provide the framework for the functioning of DeFi apps because they encode the terms and activities necessary for the functioning of these services. For example, a smart contract code has a specific code that establishes the exact terms and conditions of a loan between individuals. If certain terms or conditions are not met, collateral could be liquidated. All of this is conducted through specific code rather than manually by a bank or other institution.

All components of a decentralized finance system belong to a software stack. Each layer's components are meant to perform a specific function in the building of a DeFi system. 
Outlined below are the four layers that comprise the DeFi stack:

- Settlement Layer

- Protocol Layer

- Application Layer

- Aggregation Layer

\section{Settlement Layer}

The settlement layer is also referred to as Layer 0 because it is the base layer upon which other DeFi transactions are built. It consists of a public blockchain and its native digital currency or cryptocurrency. Transactions occurring on DeFi apps are settled using this currency, which may or may not be traded in public markets.

\section{Protocol Layer}

Software protocols are standards and rules written to govern specific tasks or activities. In parallel with real-world institutions, this would be a set of principles and rules that all participants in a given industry have agreed to follow as a prerequisite to operating in the industry.

\section{Application Layer}

As the name indicates, the application layer is where consumer-facing applications reside. These applications abstract underlying protocols into simple consumer-focused services (https://youtu.be/k9HYC0EJU6E).

\section{Aggregation Layer}

The aggregation layer consists of aggregators who connect various applications from the previous layer to provide a service to investors. For example, they might enable the seamless transfer of money between different financial instruments to maximize returns.

\section{Result}

\subsection{Decentralization}

In a centralized financial system, financial institutions are the key intermediaries mediating and controlling financial transactions. Intermediaries help reduce transaction costs, allowing financial transactions to be carried out efficiently and smoothly (Benston and Smith, 1976). As key intermediaries facilitating financial transactions, however, financial institutions can grow to dominate economic activities. When a centralized financial institution-such as Bank of America, PayPal, or Square — rises to dominance, it can accumulate disproportionate market power and profits. In a decentralized financial system, in contrast, financial transactions are facilitated not by centralized institutions but by decentralized peer-to-peer networks. When a decentralized peer-to-peer network rises to dominance, no single entity can accumulate sufficient monopoly power to monopolize the network and exclude others from participating, allowing everybody to benefit from the network effects to enlarge transaction possibilities (Davidson, 2020).

\subsection{Innovativeness}

Decentralized finance promotes permissionless and combinatorial innovation. Although a centralized platform may encourage open innovation and experimentation, its platform owners often control access and can revoke access to exert governance control. In contrast, a decentralized platform does not have a controlling party and, therefore, allows for open access and permissionless innovation - that is, developers can freely build and experiment with new applications without asking for permission. By facilitating permissionless 
innovation, decentralized platforms empower developers by guaranteeing access, allowing developers to evolve decentralized finance in organic and unexpected ways.

Decentralized platforms can also facilitate combinatorial innovation. In a decentralized finance ecosystem, new financial technologies can become the building blocks for future innovations, promoting new combinations and new products. A blockchain can be permissionless in the sense that anybody can participate in validating the blockchain, or it can be permissioned where only select parties can participate. A permissionless blockchain is usually more decentralized than a permissioned one, as power is more decentralized and diffused. In this paper, we focus on permissionless blockchains and their applications in decentralized finance. Combinatorial innovation is made possible by permissionless innovation and open sourcing in the decentralized finance ecosystem. For instance, decentralized financial applications and platforms - such as Bitcoin, Ethereum, and Libraoften publicly share their core technologies through permissive open-source licensing, allowing anybody to make use of their core technologies as well as to build new applications on top of them. In contrast, centralized financial services and platforms often carefully protect their intellectual properties through patents, copyrights, trademarks, and trade secrets, excluding others from using valuable technologies. Combinatorial innovation can accelerate the pace of financial innovation as well as increase the degree of market competition, potentially leading to newer, better, and cheaper financial services.

\subsection{Interoperability}

Decentralized finance can enhance interoperability. Traditional finance trends to work in silos, driving up transaction barriers. Different financial institutions have to maintain their own ledgers, so one financial service may not be interoperable with another. As a result, moving capital and value across silos can become costly and cumbersome. In contrast, decentralized finance is built on public blockchains and open standards, increasing the interoperability across different services. With high interoperability, financial capital and value can flow seamlessly across different services and borders, potentially creating an internet of value.

Although projects built on the same public blockchain enjoy high interoperability, decentralized finance has not achieved full interoperability yet, due to the lack of interoperability across blockchains. Entrepreneurs and innovators are exploring two potential options to achieve full interoperability. The first option is to encourage the emergence of one dominant platform and to persuade all projects to build on the same platform. Currently, Ethereum is the dominant platform for decentralized finance, and all Ethereum-based projects enjoy high interoperability.

\subsection{Borderlessness}

Centralized finance cannot be truly borderless, as it is tied to specific geographic locations with specific fiat currencies. As a result, moving capital and value across borders often encounters friction and delay. In contrast, decentralized finance is inherently borderless and thus allows for borderless finance, as it is not tied to geographic locations or fiat currencies (Gaspareniene and Remeikiene, 2020). With decentralized finance, therefore, transferring value across the globe may become as easy as sending an email, removing barriers to global value transfers. 


\subsection{Transparency}

Decentralized finance can also enhance transparency in the financial system. Centralized finance cannot have full transparency, as centralized financial institutions must secure their centralized ledgers by restricting access. Decentralized finance, in contrast, secures their public ledgers through distributed consensus and radical transparency. It records transactions on public ledgers that can be easily viewed and verified. With public ledgers, decentralized finance generates distributed trust, so transacting parties can transact with each other without pre-existing relationships or trusted intermediary, expanding the scale and scope of potential transactions (Gonzálvez-Gallego and Pérez-Cárceles, 2021). Moreover, transparent public ledgers and open-source code help keep records of all historical transactions, which can help "get to the bottom of any serious financial accident" (Kliestik et al., 2020, Siekelova et al., 2021).

\subsection{Implementation}

Blockchain technology can lead to the emergence of new business models that were previously not viable. In the financial industries, blockchain technology can reduce the involvement of centralized institutions, encourage experimentation, and broaden access to financial services. Figure 2 illustrates the rise of some business models.

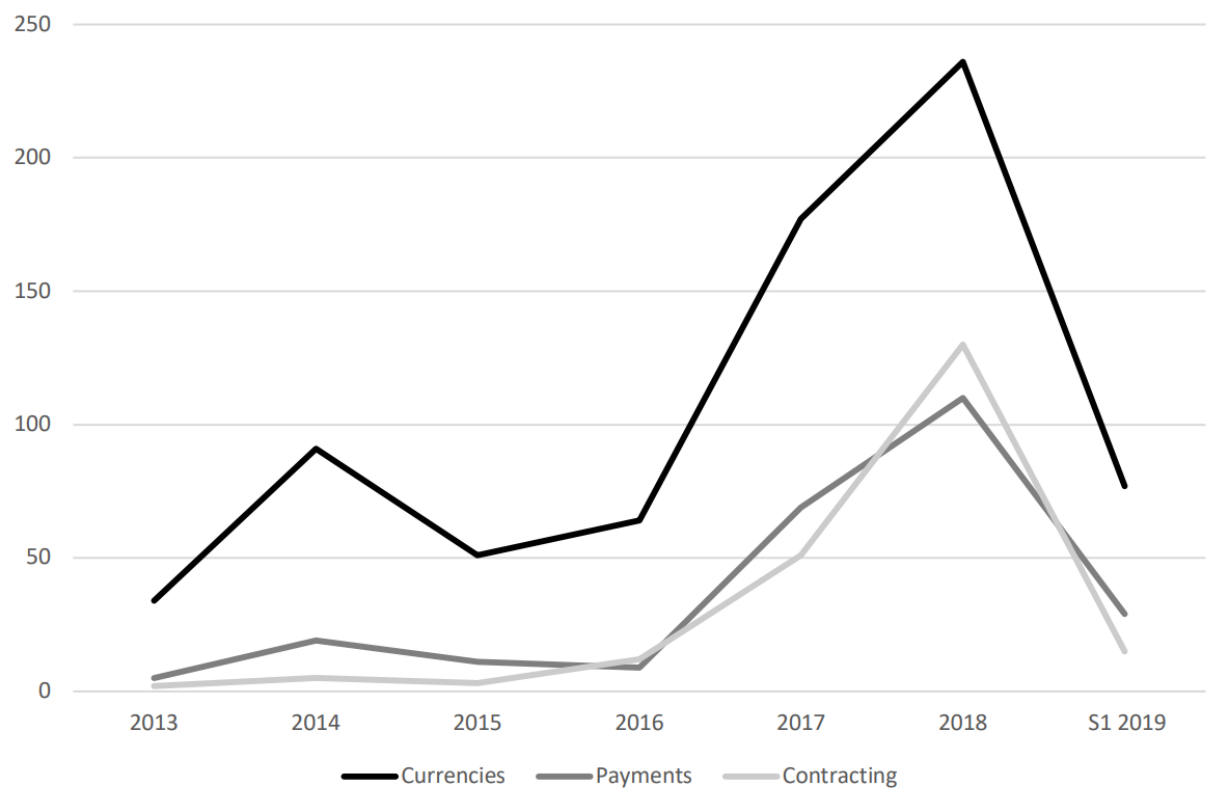

Figure 1. Number of finance-related blockchain projects across several categories

Source: Data from incheckup.com by June 2019.

Currently, decentralized currencies are the most common model, followed by contracting and payments.

\subsection{Decentralized currencies}

Bitcoin was the first decentralized cryptocurrency that was not issued by any country but rather through a decentralized technology (Nakamoto, 2008). Unlike traditional currencies issued by central banks, Bitcoin's supply schedule is fixed and cannot be changed at will, 
making it anti-inflationary. Given its decentralization, Bitcoin has become the primary store of value in the blockchain industry and is often referred to as digital gold (Kramoliš, and Dobeš 2020). Like gold, Bitcoin is inherently borderless and can be stored and transferred without the involvement of any central entity (Hu and Zhang, 2018). Besides Bitcoin, other major decentralized cryptocurrencies include Ether, Litecoin, Monero, Dash, and Zcash, among others (Tschorsch and Scheuermann, 2016).

\subsection{Decentralized payment services}

Centralized payment networks - such as Visa, PayPal, and SWIFT — facilitate online and offline commerce, but they usually charge relatively high fees for their services, especially for cross-border payments. The cost of payment services remains high, even after the internet has significantly reduced the costs of a wide variety of services.

\subsection{Decentralized fundraising}

Traditional venture financing often involves substantial friction in the fundraising process, as investors may only trust and invest in projects with strong network ties (Scott et al., 2020). Blockchain technology is reshaping the fundraising landscape (Fisch, 2019). One primary form of decentralized fundraising is an initial coin offering (ICO). In an ICO, a project would create a project-specific token on a public blockchain and sell the token to potential investors to raise funds for early-stage developments (Martino et al., 2019; Chen and Bellavitis, 2020).

\subsection{Decentralized contracting}

Contracts are essential for markets, firms, and individuals, as they facilitate collaborations and transactions. Yet, contracts can be complicated and costly. Especially, financial contracting can be hampered by adverse selection and moral hazard, raising transaction costs while restricting transaction possibilities (Feltham et al., 2016). Traditionally, transacting parties often rely on financial intermediaries to establish trust and reduce transaction costs (Benston and Smith, 1976). Over the past several years, blockchain technology has started to facilitate financial contracting by substituting financial intermediaries with smart contracts, leading to the rise of peer-to-peer financial contracting.

Smart contracts are "programs that automatically execute when pre-specified conditions (i.e., rules) in the protocols are satisfied" (Ključnikov et al., 2020).

\section{Discussion and Conclusion}

Decentralized finance is still in the beginning stages of its evolution. The total value locked in DeFi contracts is more than $\$ 41$ billion, as of March 2021. The total value locked is calculated by multiplying the number of tokens in the protocol and their value in USD. Though the total figure for DeFi may sound substantial, it is important to remember that it is notional because many DeFi tokens lack sufficient liquidity and volume to trade in crypto markets.

The DeFi ecosystem is still riddled with infrastructural mishaps and hacks. Scams also abound in the rapidly evolving DeFi infrastructure. DeFi "rug pulls" in which hackers drain a protocol of funds and investors are unable to trade, are common, though there are wellestablished protocols that can reduce this risk significantly.

The open and relatively distributed nature of the decentralized finance ecosystem might also pose problems to existing financial regulation. Current laws were crafted based on the 
idea of separate financial jurisdictions, each with its own set of laws and rules. DeFi's borderless transaction span presents important questions for this type of regulation.

Smart contracts are another area of concern for DeFi regulation. Aside from Bitcoin's success, DeFi is the clearest example of the "code is law" thesis, wherein law represents a set of rules that are written and enforced through immutable code.

For example, what if an incorrect input causes a system to crash? Or, if a compiler (which is responsible for compiling and running code) errs. Who is liable for these changes? These and many other questions need to be worked out before DeFi becomes a mainstream system used by the masses.

\section{Acknowledgements}

This research was financially supported by the Ministry of Education, Science, Research and Sport of the Slovak Republic and Slovak Academy of Sciences VEGA 1/0121/20: Research of transfer pricing system as a tool to measure the performance of national and multinational companies in the context of earnings management in conditions of the Slovak Republic and V4 countries.

\section{References}

1. Benston, G. J., \& Smith, C. W. (1976). A Transactions Cost Approach to the Theory of Financial Intermediation. Journal of Finance, American Finance Association, 31(2), 215-231.

2. Chen, I., \& Bellavitis, C. (2020). Blockchain Disruption and Decentralized Finance: The Rise of Decentralized Business Models. Journal of Business Venturing Insights, 13, eArticle 00151.

3. Davidson, R. (2020). Cyber-Physical Production Networks, Artificial Intelligence-based Decision-Making Algorithms, and Big Data-driven Innovation in Industry 4.0-based Manufacturing Systems. Economics, Management, and Financial Markets, 15(3), 1622.

4. Feltham, G. A, Hofmann, C., \& Indjejikian, R. J. (2016). Performance Aggregation and Decentralized Contracting. Accounting review, 91(1), 99-117.

5. Finematics (2020, July 2). What is DEFI? Decentralized Finance Explained [Video]. YouTube. https://www.youtube.com/watch?v=k9HYC0EJU6E

6. Fisch, Ch. (2019). Initial Coin Offerings (ICOs) to Finance New Ventures. Journal of Business Venturing, 34(1), 1-22.

7. Gaspareniene, L., \& Remeikiene, R. (2020). Arima model for predicting the development of the price of gold: European approach. Ekonomicko-manazerske spektrum. 14(1), 87-96.

8. Ginevicius, R., Kliestik, T., Stasiukynas, A., \& Suhajda, K. (2021). The Impact of National Economic Development on the Shadow Economy, Journal of Competitiveness 12(4), 39-55.

9. Gonzálvez-Gallego, N., \& Pérez-Cárceles, M.C. (2021). Does goodness of governance dissuade citizens from using cryptocurrencies?. Economics and Sociology, 14(1), 11-27.

10. Hu, K. X. \& Zhang, Z. F. (2018). Fast Lottery-Based Micropayments for Decentralized Currencies. Information security and privacy, 10946, 669-686. 
11. Kliestik, T., Valaskova, K., Lazaroiu, G., Kovacova, M., \& Vrbka, J. (2020). Remaining Financially Healthy and Competitive: The Role of Financial Predictors. Journal of Competitiveness, 12(1), 74.

12. Ključnikov, A., Civelek, M., Vozňáková, I., \& Krajčík, V. (2020). Can discounts expand local and digital currency awareness of individuals depending on their characteristics? Oeconomia Copernicana, 11(2), 239-266.

13. Kramoliš, J., \& Dobeš, K. (2020). Debt as a financial risk factor in SMEs in the Czech Republic. Equilibrium. Quarterly Journal of Economics and Economic Policy, 15(1), 87-105.

14. Kudlac, S., Majercak, J., \& Majercak, P. (2017). Comparison of different variants of logistics chain with the use of air transport using the software application. In Proceedings of 6th International Conference on Air Transport (INAIR) (pp. 45-50).

15. Majercak, P., Cisko S., \& Majercakova E. (2013). The impact of theory of constraints on the management accounting. In Proceedings of 7th International Days of Statistics and Economics (pp. 894-904).

16. Martino, P., Bellavitis, C., \& DaSilva, C. M. (2019, July 9). Blockchain and initial coin $\begin{array}{lllll}\text { offerings (ICOS): a new way of crowdfunding. } & \text { SSRN. }\end{array}$ https://ssrn.com/abstract=3414238.

17. Nakamoto, S. (2008). Bitcoin: A Peer-to-Peer Electronic Cash System. https://bitcoin.org/bitcoin.pdf

18. NBS (2015). Financial Stability Report for the year 2003-2014. National Bank of Slovakia. https://www.nbs.sk/en/publications-issued-by-the-nbs/financial-stabilityreport

19. Scott, J., Zhuravleva, N. A., Durana, P., \& Cug, J. (2020). Public Acceptance of Autonomous Vehicle Technologies: Attitudes, Behaviors, and Intentions of Users. Contemporary Readings in Law and Social Justice, 12, 23-29.

20. Siekelova, A., Belas, J., Podhorska, I., Durana, P. (2021). Accrual-Based Earnings Management: A Case Study in V4 Focusing on Mining And Quarrying Sector. Acta Montanastica, Slovaca, 26(1), 70-83.

21. Tschorsch, F., \& Scheuermann, B. (2016). IEEE Communications Surveys and Tutorials, 18(3), 2084-2123.

22. Vagner, L. (2021). Public awareness of circular economy: Case of Slovak Republic, Ekonomicko-manazerske spektrum, 15(1), 97-110. 